\title{
Evaluación de la técnica en la toma de presión arterial en un Hospital de Tercer Nivel en México
}

\author{
Evaluation of the technique for taking blood pressure \\ in a Third Level Hospital in Mexico
}

\author{
Alejandro Ezquerra Osorio, ${ }^{*}$ Eric Misael Saucedo Moreno, ${ }^{\ddagger}$ \\ Quetzalina Flores Fajardo, ${ }^{\S}$ Adriana Vergara Suárez*
}

Citar como: Ezquerra OA, Saucedo MEM, Flores FQ, Vergara SA. Evaluación de la técnica en la toma de presión arterial en un Hospital de Tercer Nivel en México. Acta Med. 2020; 18 (4): 360-366. https://dx.doi.org/10.35366/97261

\section{Resumen}

La mala técnica en la toma de presión arterial conlleva a diagnósticos y tratamientos erróneos al mostrar una presión arterial menor o mayor a la real. El objetivo es conocer cómo los profesionales de la salud aplican la técnica dentro de un hospital, y así saber si la presión arterial que reportan es la adecuada al ser comparada con un experto. Material y métodos: Estudio prospectivo, observacional y transversal que evaluó la técnica en el Hospital Ángeles Mocel. Se observaron 101 participantes, incluyendo personal de enfermería, médicos residentes, internos de pregrado y auxiliares de enfermería. Resultados: Las omisiones más frecuentes fueron falta de cuestionamiento por vejiga vacía en $99 \%$ y no preguntar si recibió medicamentos simpaticomiméticos en $96 \%$. Únicamente $4 \%$ tuvieron técnica aceptable, siguiendo ocho o más pasos de los 12 establecidos. No se identificó diferencia en la calidad de la técnica de acuerdo con el cargo entre médicos o enfermería ( $p=0.113$ ). Se obtuvo diferencia significativa en la presión diastólica del grupo de técnica aceptable frente a inadecuada $(p=0.020)$ y regular contra inadecuada $(p=0.041)$. Conclusión: Dentro del hospital no se siguen los pasos adecuados en la medición de presión arterial. Dada una mejor técnica, la variación de la diastólica es menor.

Palabras clave: Hipertensión, baumanómetro, guías AHA, presión arterial.

\section{Abstract}

The poor technique in measuring blood pressure leads to misdiagnosis and an incorrect treatment by showing higher or lower blood pressure than the actual. The objective is to understand how health professionals adhere to the technique within a hospital, and verify if the blood pressure reported is adequate when compared to an expert. Material and methods: A prospective, observational, cross-sectional study was performed and the technique was evaluated at the Mocel Ángeles Hospital. 101 participants were observed, including nurses, residents, interns and nursing assistants. Results: The most frequent omissions were not asking about empty bladder ( $99 \%$ of the cases) and not asking whether the patient received sympathomimetic medications in $96 \%$. Only $4 \%$ presented an acceptable technique with more than eight of the 12 steps. There was no identified difference in the quality of the technique according to the position between doctors or nurses $(p=0.113)$. We observed a significant difference in diastolic pressure between the acceptable technique group and the inadequate technique group $(p=0.020)$ and between regular and inadequate $(p=0.041)$. Conclusion: Health professionals do not follow the right procedure when measuring blood pressure inside the hospital. Given a better technique, the variation in diastolic is smaller.

Keywords: Hypertension, baumanometer, AHA guidelines, blood pressure.

\section{www. medigraphic. org. mx}

\footnotetext{
* Residente de Medicina Interna, Facultad Mexicana de Medicina, Universidad La Salle México.

₹ Servicio de Cirugía General, Hospital Ángeles Mocel.

§ Servicio de Medicina Interna, Hospital Ángeles Mocel.
}

Correspondencia:

Dr. Alejandro Ezquerra Osorio

Correo electrónico: aeo900@yahoo.com.mx

Aceptado: 04-02-2020.

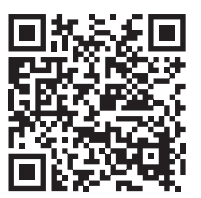




\section{INTRODUCCIÓN}

De acuerdo con la Encuesta Nacional de Salud y Nutrición (ENSANUT) 2016, la prevalencia de hipertensión arterial en México es de 25.5\%; es decir, uno de cada cuatro adultos padece hipertensión arterial sistémica. ${ }^{1}$ La toma de presión arterial (PA) es una técnica necesaria para el diagnóstico y seguimiento de la hipertensión arterial, así como la toma de decisiones en otras patologías.

En 1856 se obtuvo la primera determinación directa de la PA en el humano. En 1881 se utilizó la columna de mercurio para la medición de PA. ${ }^{2}$ En el siglo XXI, los profesionales de la salud no toman la PA de forma adecuada y a pesar de tener aparatos más sofisticados existen variaciones interobservador. ${ }^{3,4}$ Esto es un problema, pues la técnica incorrecta de PA puede sobrediagnosticar de 20 a 30\% de la población con hipertensión arterial, lo que resulta en tratamiento inapropiado. ${ }^{5}$

Múltiples sociedades internacionales han publicado directrices para la determinación de la PA, la American Heart Association (AHA), ${ }^{6}$ en el 2017, publicó las Guías para la prevención, detección, evaluación y manejo de la hipertensión arterial en adultos, donde describen la técnica para la toma de la PA.

Las dos guías más actuales de hipertensión arterial sistémica son la American College of Cardiology (ACC) junto con la AHA en 2017 y la European Society of Cardiology (ESC). La definición y clasificación de hipertensión arterial ha cambiado con el tiempo y esto varía entre las diferentes guías. De acuerdo con la guía americana, se define "hipertensión" con 130/80 versus 140/90 mmHg de la europea. ${ }^{7}$

El baumanómetro de mercurio es el estándar de oro para la toma de PA; por seguridad, ya casi no se utiliza y se prefieren métodos electrónicos o manuales con baumanómetros aneroides, la diferencia es mínima y lo importante es la técnica. ${ }^{8}$

En el año 2017, la ACC/AHA nuevamente publicó el método para la toma de la PA. ¿Por qué volver a enfatizar en la técnica? Un estudio realizado a 250 enfermeras en Irán mostró que aun cuando conocían la teoría sobre la técnica en la toma de PA, esto no garantizaba un adecuado procedimiento. $^{9}$

Hay factores que modifican la presión sistólica y diastólica durante su toma. Por ejemplo, la presión sistólica aumenta hasta $17 \mathrm{mmHg}$ cuando se habla, $2 \mathrm{mmHg}$ si no tiene soporte el brazo, disminuye $8 \mathrm{mmHg}$ con un brazalete pequeño y aumenta $8 \mathrm{mmHg}$ la diastólica; ${ }^{5}$ entonces, un paciente que llegue con presión sistólica de $80 \mathrm{mmHg}$ a urgencias, podría tener $100 \mathrm{mmHg}$ si se mide con técnica inadecuada. La ACC/AHA recomienda reposo de 30 minutos posterior a realizar actividades físicas. La PA sistólica aumenta de acuerdo con el tipo de actividades, por ejemplo, durante una reunión con personas se incrementa $20 \mathrm{mmHg}$, trabajar $16 \mathrm{mmHg}$, caminar $14 \mathrm{mmHg}$, vestirse $11 \mathrm{mmHg}$, comer $8 \mathrm{mmHg}$, leer $1.9 \mathrm{mmHg}$ y ver televisión $0.3 \mathrm{mmHg} .{ }^{10}$

Una inadecuada técnica puede diagnosticar mal a un paciente con hipotensión ortostática, causa frecuente de visita a urgencias. En San Diego, California, se realizó un estudio que abarcó 100 pacientes hospitalizados, en el cual se demostró que la posición del brazo en forma perpendicular al cuerpo cuando se toma la PA, disminuye la presión sistólica y diastólica, esté la persona en bipedestación o supino versus el brazo paralelo. ${ }^{11}$

Es frecuente que el personal de salud, dentro y fuera del hospital, realice una técnica inadecuada al tomar la PA y esto es un problema global. En un estudio por Villegas se evaluó en el hospital a 113 médicos y 56 enfermeras y se descubrió que ninguno cumplía todos los pasos en la toma de la PA. ${ }^{12}$ En India se evaluó dentro del hospital a 400 profesionales de la salud, incluidos médicos, paramédicos y enfermeras, lo que demostró que $1.43 \%$ de médicos y $0.5 \%$ de enfermería tenían excelente técnica. ${ }^{13}$ En una revisión de Estados Unidos, de 114 médicos evaluados, $96 \%$ no esperaron el periodo de reposo y $77 \%$ no tomaron la PA en ambos brazos. ${ }^{14}$ En España, un estudio aplicado a personal de enfermería en pacientes hospitalizados, encontró diferencia de $5 \mathrm{mmHg}$ en la PA sistólica $\left(\mathrm{IC}_{95 \%}\right.$, 3-6 $\mathrm{mmHg}$ y $\mathrm{p}$ menor 0.01 ) entre el investigado y la correcta toma del investigador. ${ }^{2}$

A diferencia de pacientes ambulatorios, se han realizado pocos estudios en la técnica para la toma de PA en pacientes hospitalizados. La correcta técnica garantiza un valor adecuado de PA, lo que permitirá diagnosticar o tratar oportunamente pacientes hospitalizados con hipotensión ortostática, emergencia hipertensiva, iniciar vasopresores en choque séptico, modificar antihipertensivos de forma segura, o cualquier otra entidad donde el valor de presión arterial es fundamental para la toma de decisiones.

Considerando que en otros países se toma mal la PA en pacientes hospitalizados, es importante conocer la situación en México. Nuestro estudio busca conocer si existen errores en la técnica de toma de PA por profesionales de la salud, en pacientes internados en un hospital privado de la Ciudad de México, y si existen variaciones interobservador entre los evaluados contra las de un evaluador al seguir todos los pasos de la técnica.

\section{MATERIAL Y MÉTODOS}

Se realizó un estudio prospectivo, observacional, transversal, donde se evaluó un Hospital de Tercer Nivel de la Ciudad de México en el sector privado la técnica para 
tomar la presión arterial por método auscultatorio, de acuerdo con la Guía ACC/AHA 2017. Se elaboró una lista de verificación con base en los estándares de la técnica que menciona la guía, la cual consistió en 12 pasos. Para las mediciones de PA se utilizó un baumanómetro aneroides de pedestal marca Welch Allyn modelo WA7670-10, calibrado por personal de biomédica del hospital al menos tres meses previos. Las mediciones de PA fueron en pacientes hospitalizados de forma aleatoria, con edades de 18 a 90 años en las Áreas de Medicina Crítica, Medicina Interna, Cirugía y Urgencias. A los participantes se les clasificó de acuerdo con el cargo que tienen dentro del hospital. Todos fueron evaluados por un solo evaluador, con el mismo test, mientras realizaban la toma de PA en pacientes, se les explicó que se calificaría la técnica. Se excluyeron residentes de radiología y enfermeras de pediatría.

De acuerdo con los pasos realizados de manera correcta en la técnica, se clasificaron en tres grupos. Grupo 1: inadecuada técnica, aquellos con cuatro pasos o menos realizados. Grupo 2: regular técnica, con cinco a ocho pasos realizados. Grupo 3: aceptable técnica, aquellos con más de nueve pasos. Para buscar diferencias con los tres grupos en la calidad de la técnica y poder comparar de manera objetiva las presiones sistólicas y diastólicas de los evaluados versus las presiones de nuestro estándar, utilizamos la prueba de Kruskal-Wallis.

Se consideró como toma estándar aquélla que fue tomada por un solo evaluador, que siguió todos los pasos de la técnica y que desconoce los datos tomados por el grupo evaluado. Se calculó sensibilidad y especificidad, así como la razón de momios del tipo de técnica.

El análisis estadístico se realizó con el Software SPSS versión 20.0 (SPSS, Chicago, EUA); mientras que para datos categóricos la prueba exacta de Fisher. La prueba t de Student para datos pareados para comparar la media entre grupos, se consideró un $\mathrm{p}=0.05$ con un $\mathrm{IC}_{95 \%}$.

\section{RESULTADOS}

Se evaluaron 101 participantes, se les clasificó de acuerdo con el cargo que tienen dentro del hospital, de los cuales, 48 representan a enfermería, 30 residentes, 13 médicos internos de pregrado, siete auxiliares de enfermería como otros y tres pasantes de enfermería.

Al clasificarse en tres grupos de acuerdo con la técnica evaluada, se encontró que $67 \%$ tiene una técnica regular, $28 \%$ inadecuada técnica y sólo $4 \%$ técnica aceptable, ninguno cumplió los 12 puntos de la técnica. De las 48 enfermeras, ninguna demostró técnica aceptable; mientras que de 30 residentes, dos mostraron técnica aceptable. Al comparar las proporciones de los grupos de acuerdo con el cargo, al utilizar la prueba exacta de Fisher, encontramos un resultado de $p=0.113$, por lo que interpretamos que no existe diferencia en la técnica de toma de presión arterial de acuerdo con el cargo, ya que la gran mayoría tienen técnica regular (Tabla 1).

En la Tabla 2 se representan los puntos evaluados de acuerdo con cada cargo, sin encontrar diferencias significativas entre ellos. Los errores más frecuentes fueron: no preguntar por vejiga vacía, no confirmar que el paciente haya recibido medicamentos tipo simpaticomiméticos o anticolinérgicos, así como rehabilitación física previa, no preguntar por el brazo con la presión arterial más elevada o, en su defecto, tomarla en ambos brazos para conocerlo, sólo lo realizaron 1, 4 y 9\% respectivamente de los 101 evaluados. Lo que la mayoría realizó fue escoger el tamaño correcto del brazalete en $96 \%$, remover la ropa del brazo el $92 \%$ y sorprende que no todos informan al paciente el valor de la PA, sólo un $87 \%$.

Pudimos observar que, al comparar los grupos por separado con el estándar de oro, existen diferencias significativas en las medias de presiones sistólica y diastólica, con $\mathrm{p}=0.010\left(\mathrm{IC}_{95 \%}[0.710\right.$ a 5.092$\left.]\right)$ y $\mathrm{p}=0.005\left(\mathrm{IC}_{95 \%}\right.$ [3.371 a 2.860]), respectivamente. Al comparar los grupos

\begin{tabular}{lcccc} 
& \multicolumn{2}{c}{ Tabla 1: Evaluación por cargo y calidad de la técnica. } \\
& $n(\%)$ & Técnica inadecuada $n$ (\%) & Técnica regular $n$ (\%) & Técnica aceptable $n(\%)$ \\
\hline Pasante & $3(5.0)$ & 0 & $2(2.9)$ & $1(25.0)$ \\
Enfermería & $48(47.5)$ & $18(62.1)$ & $30(44.1)$ & 0 \\
MIP & $13(12.9)$ & $2(6.9)$ & $10(14.7)$ & $1(25.0)$ \\
Residente & $30(29.7)$ & $8(27.6)$ & $6(29.4)$ & $2(50.0)$ \\
Otros & $7(6.9)$ & $1(3.4)$ & $68(67.5)$ & 0 \\
Total & $101(100.0)$ & $29(28.7)$ & & $4(3.8)$ \\
\hline MIP = Médicos internos de pregrado. & & &
\end{tabular}


Tabla 2: Porcentaje de realización de los pasos de acuerdo con los diferentes cargos.

\begin{tabular}{|c|c|c|c|c|c|c|}
\hline & $\begin{array}{c}\text { Pasante } \\
\text { enfermería } \\
(n=3) \\
n(\%)\end{array}$ & $\begin{array}{l}\text { Enfermería } \\
\qquad(\mathrm{n}=48) \\
\mathrm{n}(\%)\end{array}$ & $\begin{array}{c}\text { MIP } \\
(n=13) \\
n(\%)\end{array}$ & $\begin{array}{c}\text { Residente } \\
(n=30) \\
n(\%)\end{array}$ & $\begin{array}{c}\text { Otros } \\
(n=7) \\
n(\%)\end{array}$ & $p$ \\
\hline Paciente relajado, sentado & $1(7.7)$ & $5(38.5)$ & $3(23.2)$ & $4(30.8)$ & 0 & 0.632 \\
\hline $\begin{array}{l}\text { ¿Preguntó por ejercicio o } \\
\text { medicamentos previos? }\end{array}$ & $1(20.0)$ & $1(20.0)$ & 0 & $3(60.0)$ & 0 & 0.619 \\
\hline ¿Preguntó por vejiga vacía? & 0 & $1(100.0)$ & 0 & 0 & 0 & 0.471 \\
\hline $\begin{array}{l}\text { Paciente o evaluador hablan } \\
\text { durante procedimiento }\end{array}$ & $3(7.3)$ & $22(53.7)$ & $5(12.2)$ & 7 (17.1) & $4(9.8)$ & 0.087 \\
\hline Sí retiró la ropa del brazo & $3(3.3)$ & $40(43.5)$ & $13(14.1)$ & $29(31.5)$ & $7(7.6)$ & 0.056 \\
\hline $\begin{array}{l}\text { No conoce la calibración del } \\
\text { baumanómetro }\end{array}$ & $3(3.0)$ & $48(47.5)$ & $13(12.9)$ & $3(29.7)$ & $7(6.9)$ & 1.010 \\
\hline ¿Colocó soporte en el brazo? & $2(3.6)$ & $29(51.8)$ & $7(12.5)$ & $15(6.8)$ & $3(5.4)$ & 0.242 \\
\hline $\begin{array}{l}\text { ¿Colocó el brazalete a la altura } \\
\text { del corazón? }\end{array}$ & $3(3.9)$ & $34(44.7)$ & $11(14.5)$ & $2(30.3)$ & $3(3.9)$ & 0.918 \\
\hline $\begin{array}{l}\text { ¿Tamaño apropiado del } \\
\text { brazalete? }\end{array}$ & $3(3.1)$ & $45(46.9)$ & $13(13.5)$ & $29(30.2)$ & $6(6.3)$ & 0.843 \\
\hline $\begin{array}{l}\text { ¿Preguntó por el brazo con la } \\
\text { presión arterial más elevada? }\end{array}$ & $1(11.1)$ & $5(55.6)$ & $1(11.1)$ & $2(22.2)$ & 0 & 0.219 \\
\hline ¿Palpó el pulso radial? & 0 & $1(11.1)$ & $4(44.4)$ & $4(44.4)$ & 0 & 0.296 \\
\hline $\begin{array}{l}\text { ¿Desinfló a } 2 \text { mmHg por } \\
\text { segundo? }\end{array}$ & $3(4.8)$ & $28(44.4)$ & $7(11.1)$ & $21(33.3)$ & $4(6.3)$ & 0.673 \\
\hline $\begin{array}{l}\text { ¿Informó al paciente la cifra de } \\
\text { presión arterial? }\end{array}$ & $3(3.4)$ & $37(42.5)$ & $12(13.8)$ & 29 (33.3) & $6(6.9)$ & 0.068 \\
\hline
\end{tabular}

obtuvimos una diferencia significativa en el grupo 1 (mala técnica) y el grupo 2 (regular técnica), con lo que podemos interpretar que existen variaciones clínicamente importantes en la toma de presión, de acuerdo con la calidad de la técnica, lo cual es comprobable al no obtener esa misma diferencia en el grupo con técnica aceptable (Tabla 3).

Las Figuras 1 y 2, representan de forma gráfica los resultados obtenidos en la Tabla 3. Al comparar las medias de la presión sistólica y diastólica obtenida por el grupo estudiado o control (azul) versus la media de presión sistólica y diastólica del estándar de oro (rojo), identificamos diferencias significativas en las medias para la presión sistólica en mala y regular técnica.

Calculamos con cuadros de contingencia la sensibilidad y especificidad de la prueba de acuerdo con la calidad de la toma, encontrando para técnica aceptable una sensibilidad de $66.7 \%\left(\mathrm{IC}_{95 \%}\right.$ [35.4 a $\left.\left.87.9 \%\right]\right)$ y especificidad de $83.1 \%$ $\left(\mathrm{IC}_{95 \%}[71.5\right.$ a $\left.90.5 \%]\right)$ con una razón de momios para ser diagnosticados con hipertensión arterial de 9.8 sobre una técnica estándar.

\section{DISCUSIÓN}

En Italia evaluaron al personal médico y de enfermería en 14 hospitales, sólo 1.4\% de 1,334 evaluados midió la circunferencia del brazo para escoger el brazalete correcto, $28 \%$ preguntó si había ingerido cafeína o realizado algún esfuerzo previo. En cambio, las acciones que la mayoría realizó fue que el paciente se mantuvo en silencio y con brazo descubierto en 92 y $94 \%$ de los casos, respectivamente. ${ }^{3}$ En nuestro estudio, sólo $47 \%$ de pacientes y evaluados mantuvieron silencio, de forma similar con el estudio italiano el 92\% removió ropa del brazo.

Un brazalete de tamaño menor a la circunferencia del brazo sobrestima la PA, mientras que uno mayor al tamaño de la circunferencia del brazo subestima PA. ${ }^{15}$ Esto suele ser 
Tabla 3: Prueba t de Student para datos emparejados entre el grupo evaluado y el estándar de oro.

\begin{tabular}{|c|c|c|c|c|}
\hline & & Estándar de oro* & $\mathrm{p}$ & $\mathrm{IC}_{95 \%}$ \\
\hline \multicolumn{5}{|l|}{ Grupo evaluado total* } \\
\hline Presión sistólica & $109.54 \pm 12.993$ & $106.64 \pm 16.150$ & 0.010 & 0.710 a 5.092 \\
\hline Presión diastólica & $69.07 \pm 9.746)$ & $67.08 \pm 7.914$ & 0.005 & 3.371 a 2.860 \\
\hline \multicolumn{5}{|l|}{ Grupo técnica mala* } \\
\hline Presión sistólica & $109.59 \pm 14.85$ & $105.5 \pm 16.449$ & 0.044 & -2.92 a 6.23 \\
\hline Presión diastólica & $66.55 \pm 9.187$ & $66.03 \pm 7.835$ & 0.050 & 0.001 a 5.033 \\
\hline \multicolumn{5}{|l|}{ Grupo técnica regular* } \\
\hline Presión sistólica & $109.97 \pm 11.562$ & $106.46 \pm 15.579$ & 0.010 & 6.163 a 2.649 \\
\hline Presión diastólica & $69.79 \pm 9.897$ & $67.13 \pm 7.596$ & 0.045 & -0.105 a 3.428 \\
\hline \multicolumn{5}{|c|}{ Grupo técnica aceptable* } \\
\hline Presión sistólica & $116.50 \pm 21.810$ & $115.00 \pm 25.495$ & 0.706 & 13.011 a 0.415 \\
\hline Presión diastólica & $77.50 \pm 9.574$ & $73.75 \pm 12.500$ & 0.215 & -3.865 a 11.367 \\
\hline
\end{tabular}

Figura 1: Comparación de la media de presión sistólica entre el grupo control versus el estándar, de acuerdo con la técnica utilizada (mala, regular o aceptable).

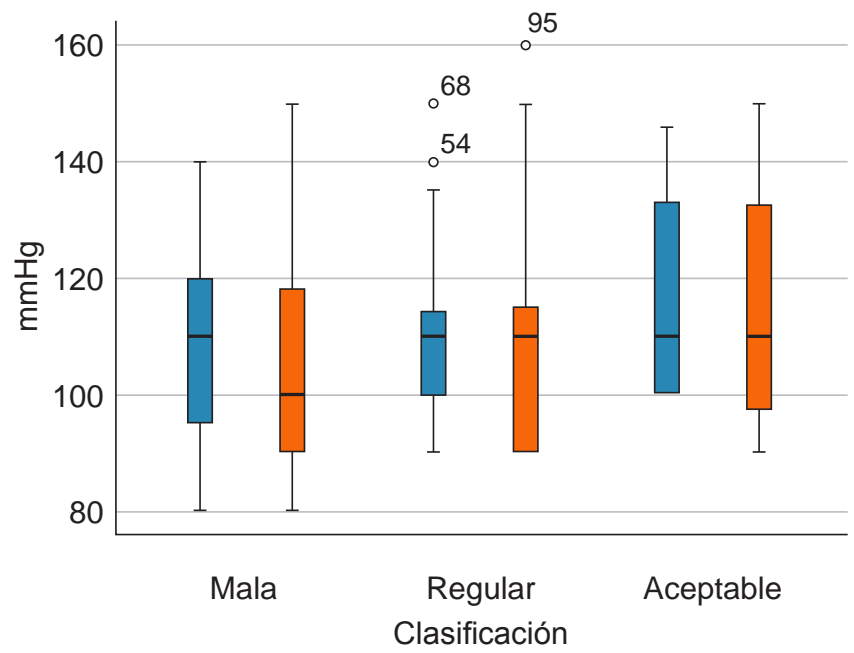

Sistólica evaluado
Figura 2: Comparación de la media de presión diastólica entre el grupo control versus el estándar, de acuerdo con la técnica utilizada (mala, regular o aceptable).

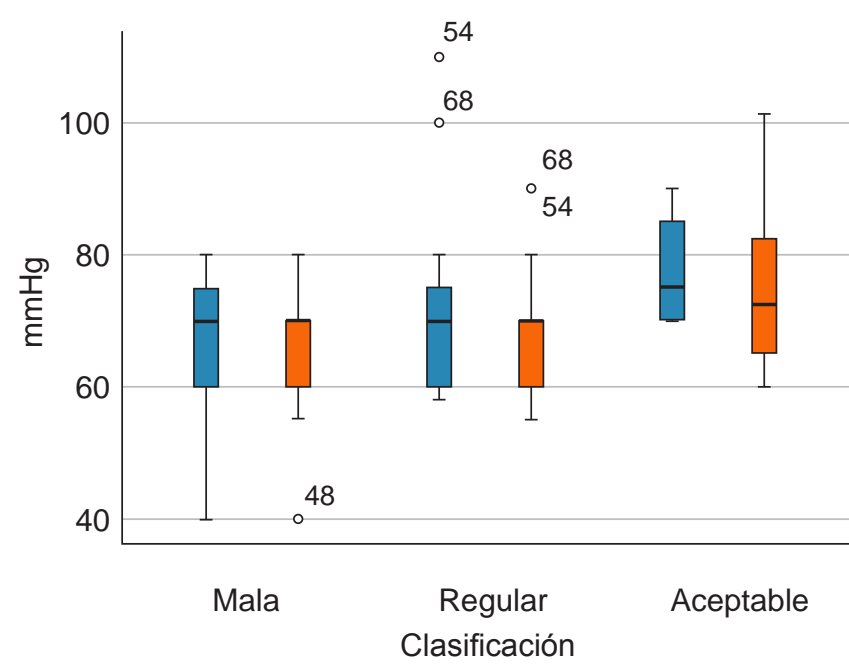

Diastólica evaluado

Diastólica estándar un error frecuente, pero en nuestro estudio, 96\% escogió el brazalete de tamaño apropiado, una de las razones que pudo influenciar es que en los pacientes no hubo quien tuviera obesidad o bajo peso, a pesar de escogerse de manera aleatoria.

Un estudio realizado en el Hospital de Tercer Nivel de Nigeria evaluaron a 103 médicos y 191 enfermeras en la toma de PA con pacientes hospitalizados, en éste se demostró que las enfermeras tienen mejor conocimiento y técnica que los médicos. ${ }^{16}$ Esto contrasta con nuestro estudio, donde dos médicos residentes lograron una técnica aceptable, mientras que ninguna enfermera entró en esta categoría. Respecto a la técnica regular, 66\% de los médicos residentes mostraron este nivel de técnica versus $62 \%$ de enfermería.

Dos evaluados no utilizaron estetoscopio y se guiaron por el pulso radial o aguja del baumanómetro, el resto utilizó el diafragma del estetoscopio, nadie la campana. En 1999, la OMS recomendó utilizar la campana, pero un estudio en Finlandia demostró que se puede utilizar la 
campana o diafragma del estetoscopio, pero es más factible el diafragma, porque cubre más área y es fácil de sostener. ${ }^{17}$

La velocidad de inflado del brazalete no tiene efecto en la presión arterial, pero si el desinflado. Desinflar a menos de $2 \mathrm{mmHg}$ por segundo disminuye los ruidos de Korotkoff, lo que resulta en aumento de la presión diastólica, ${ }^{18}$ por lo tanto, infraestima la presión sistólica y sobrestima la diastólica. ${ }^{19}$ En nuestro estudio, sólo $63 \%$ desinfló el brazalete a la velocidad adecuada.

La calibración se debe realizar cada seis meses, ya que las piezas de metal en baumanómetros aneroides se suelen aflojar. En promedio, 30 a 40\% de baumanómetros aneroides están descalibrados $4 \mathrm{mmHg}$ y $10 \%$ hasta 10 $\mathrm{mmHg} .{ }^{10} \mathrm{El} 74 \%$ de nuestra población desconocía cuándo se realizó la calibración del aparato y cada cuánto se lleva a cabo en el hospital.

Siempre se tomará el valor de PA del brazo donde inicialmente se encontró más elevada; en caso de no conocerse, se deberá hacer la medición en ambos brazos. ${ }^{5}$ Un metaanálisis demostró mayor riesgo de mortalidad en enfermedades vasculares, con diferencia mayor a $10 \mathrm{mmHg}$ entre ambos brazos. ${ }^{20}$ Sólo $9 \%$ de los evaluados preguntaron si el paciente tenía hipertensión arterial sistémica y conocía en qué brazo se debía de medir, ninguno del estudio tomó la presión arterial en ambos brazos.

En nuestro estudio, pudimos comprobar estadísticamente que un hay diferencia en la PA diastólica de acuerdo con la calidad de la técnica, no así para la PA sistólica; no obstante, clínicamente sí encontramos diferencias significativas, con mayor tendencia a obtener menor variabilidad de la toma de presión con una mejor técnica. Este hallazgo es de suma relevancia, porque tan sólo $5 \mathrm{mmHg}$ de diferencia, diastólica 89 vs $94 \mathrm{mmHg}$, reduce $62 \%$ de los pacientes con hipertensión e infradiagnostican hipertensión arterial, retrasando el tratamiento a 21 millones de personas en Estado Unidos. ${ }^{10}$ En el lado opuesto, $5 \mathrm{mmHg}$ hacia arriba diagnosticará el doble de pacientes con hipertensión arterial, duplicándolo a 27 millones de personas las cuales recibirán tratamiento sin necesitarlo,, 21 incrementará los costos de salud pública y puede ocasionar efectos secundarios de las medicinas en la persona. ${ }^{22}$ También impacta en las comorbilidades, la reducción $2 \mathrm{mmHg}$ de la presión diastólica disminuye 15\% el riesgo de evento vascular cerebral. ${ }^{23}$

Parte de este problema en la técnica de la presión arterial viene desde la universidad. En Madrid, evaluaron a estudiantes de tercer y sexto año de medicina en la Universidad Autónoma sobre la toma de PA; sólo $28.6 \%$ de tercer año y $61.9 \%$ de sexto año saben cómo tomar correctamente la PA, sólo $71 \%$ de sexto año han entrenado de forma práctica la toma de PA. ${ }^{24}$

Valores de PA sistólica y diastólica con inadecuada técnica pueden subdiagnosticar hipertensión arterial y no recibir tratamiento en pacientes ambulatorios. En cambio, en pacientes hospitalizados, valores de PA mal tomados pueden significar cargas o bolos de soluciones o iniciar vasopresores para elevar la PA, o al revés, aumentar dosis de antihipertensivos con posibles efectos secundarios. Por lo cual, este estudio realizado en pacientes hospitalizados a profesionales de la salud, busca concientizar el impacto de una inadecuada técnica.

¿Qué pasaría si se mide correctamente la técnica en pacientes hospitalizados? Por pensar otros ejemplos, ¿se tratará oportunamente con soluciones y vasopresor un choque séptico? ¿Se trombolizará un evento vascular cerebral isquémico de forma más segura? ¿Debemos seguir confiando en las presiones arteriales que nos reporta el personal hospitalario o comenzar a verificar nosotros la PA para tomar nuestra decisión del tratamiento?

Una de las grandes deficiencias de nuestro estudio es, sin duda alguna, el número de participantes evaluados, seguramente con una mayor muestra obtendríamos valores estadísticamente significativos para la toma de presión sistólica. Todos los estudios previos y la técnica estándar se han realizado con baumanómetros aneroides, decidimos apegarnos a la evidencia y realizarlo con aneroides sabiendo que en la mayoría de los hospitales y en el nuestro la presión arterial se mide con baumanómetros electrónicos, siendo ésta una limitante.

\section{CONCLUSIÓN}

La toma de PA se ha convertido en un proceso mecánico del día a día, donde se saltan muchos pasos en su medición. Este estudio demuestra que los profesionales de la salud miden incorrectamente la presión arterial en el hospital sin importar sin son médicos o personal de enfermería, por lo tanto, en pacientes internados se realiza diagnóstico erróneo o tratamiento inapropiado. El diagnóstico de hipertensión arterial y su ajuste del tratamiento, diagnóstico de hipotensión ortostática o la decisión de trombolizar un evento vascular cerebral de acuerdo con la presión arterial pueden estar decidiéndose con valores obtenidos de forma incorrecta y, por ende, diagnósticos y tratamientos erróneos. Esperamos que este estudio busque motivar a los profesionales de la salud a seguir el protocolo en la toma de la presión arterial y a revisar la calibración de sus aparatos que, sin duda, a mediano o lejano plazo ahorrará costos en medicinas y complicaciones relacionadas con la presión arterial con gran impacto en la salud pública.

\section{REFERENCIAS}

1. Instituto Nacional de Salud Pública. Encuesta Nacional de Salud y Nutrición de Medio Camino 2016 Informe Final de Resultados. [Internet]. Gob.mx. 2019 [Citado 18 Mayo 2019]. Disponible 
en: https://www.gob.mx/cms/uploads/attachment/file/209093/ ENSANUT.pdf.

2. García M, Samblás D, Estéves P, Hernández G, Márquez Correa. El paradigma de la tensión arterial medida por los profesionales de enfermería. Enferm Cardiol. 2006; 12 (39): 13-15.

3. Manzoli L, Simonetti V, D'Errico M, De Vito C, Flacco M, Forni C et al. (In)accuracy of blood pressure measurement in 14 Italian hospitals. Am J Hypertens. 2012; 30 (10): 1955-1960.

4. Graves JW, Sheps SG. Does evidence based medicine suggest that physicians should not be measuring blood pressure in the hypertensive patients? Am J Hypertens. 2004; 17: 354e360.

5. McAlister F. Evidence based treatment of hypertension: measurement of blood pressure: an evidence based review. Br Med J. 2001; 322 (7291): 908-911.

6. 2017 ACC/AHA/AAPA/ABC/ACPM/AGS/APhA/ASH/ASPC/NMA/ PCNA Guideline for the prevention, detection, evaluation, and management of high blood pressure in adults: a report of the American College of Cardiology/American Heart Association Task Force on Clinical Practice Guidelines. J Am Coll Cardiol. 2018; 71: e127-e248.

7. Williams B, Mancia G, Spiering W, Agabiti RE, Azizi M, Burnier $M$ et al. 2018 ESC/ESH Guidelines for the management of arterial hypertension. Eur Heart J. 2018; 39 (33): 3021-3104.

8. Mlawanda G, Pather M, Govender S. An analysis of blood pressure measurement in a primary care hospital in Swaziland. Afr J Prim Health Care Fam Med. 2014; 6 (1): 590.

9. Dokoohaki R, Raeiskarimian F, Rahgosha A, Sharifi M. The frequency of errors of blood pressure measurement among nurses in the hospital affiliated to Shiraz University of Medical Sciences. Int Cardiovasc Res J. 2015; 9 (1): 41-45.

10. Campbell N, McKay D. Accurate blood pressure measurement: why does it matter? CMAJ. 1999; (3): 277-278.

11. Guss D, Abdelnur D, Hemingway T. The impact of arm position on the measurement of orthostatic blood pressure. J Emerg Med. 2008; 34 (4): 377-382.

12. Villegas I, Arias IC, Botero A, Escobar A. Evaluation of the technique used by health-care workers for taking blood pressure. Hypertension. 1995; 26: 1204-1206.
13. Mohan B, Aslam N, Ralhan U, Sharma S, Gupta N, Singh V et al. Office blood pressure measurement practices among community health providers (medical and paramedical) in northern district of India. Indian Heart J. 2014; 66 (4): 401-407.

14. McKay DW, Campbell NRC, Parab LS, Chockalingam A, Fodor JG. Clinical assessment of blood pressure. J Hum Hypertens. 1990; 4: 639645.

15. Jones D, Appel L, Sheps S, Roccella E, Lenfant C. Measuring blood pressure accurately. JAMA. 2003; 289 (8): 1027-1031.

16. Ojo O, Sogunle P, Malomo S, Adeyemo A. Knowledge of blood pressure measurement among tertiary hospital staff in south west Nigeria and its related sociodemographic determinants. J Family Med Community Health. 2018; 5 (3): 1153-1167.

17. Kantola I, Vesalainen R, Kangassalo K, Kariluoto A. Bell or diaphragm in the measurement of blood pressure? Am J Hypertens. 2005; 23 (3): 499-503.

18. Ogedegbe G, Pickering T. Principles and techniques of blood pressure measurement. Cardiology Clin. 2010; 28 (4): 571-586.

19. Chobanian AV, Bakris GL, Black HR, Cushman WC, Green LA, Izzo JL et al. The seventh report of the joint national committee on prevention, detection, evaluation, and treatment of high blood pressure: the JNC 7 complete report. Hypertension. 2003; 42: 1206e1252.

20. Clarck C, Taylor RS, Shore AC, Ukoumunne OC, Campbell JL. Association of a difference in systolic blood pressure between arms with vascular disease and mortality: a systematic review and metaanalysis. Lancet. 2012; 379: 905-914.

21. Mitka M. Many physician practices fall short on accurate blood pressure measurement. JAMA. 2008; 299 (24): 2842-2849.

22. Joffres MR, Hamet P, Rabkin SW, Gelskey D, Hogan K, Fodor G. Prevalence, control and awareness of high blood pressure among Canadian adults. CMAJ•JAMC. 1992; 146 (11): 1997-2005.

23. Ray G, Nawarskas J, Anderson J. Blood pressure monitoring technique impacts hypertension treatment. J Gen Intern Med. 2011; 27 (6): 623-629.

24. González-López J, Ramírez J, García R, Esteban S, del Barrio J, Rodríguez-Artalejo F. Knowledge of correct blood pressure measurement procedures among medical and nursing students. Rev Esp Cardiol. 2009; 62 (5): 568-571. 